\title{
Correction to: A brief intervention for weight control based on habit- formation theory delivered through primary care: results from a randomised controlled trial
}

\author{
R. J. Beeken · B. Leurent $\cdot$ V. Vickerstaff $\cdot$ R. Wilson $\cdot$ H. Croker $\cdot$ S. Morris $\cdot$ R. Z. Omar $\cdot$ I. Nazareth $\cdot$ J. Wardle
}

Published online: 7 June 2021

(c) The Author(s) 2021. This article is published with open access

Correction to: International Journal of Obesity

https://doi.org/10.1038/ijo.2016.206

The original version of this article unfortunately contained a mistake in Table 1. In the paper the authors report cholesterol as mg dl-1 (Table 1) however, the correct unit should be mmol/l. Glucose should be the same. The authors apologize for the error. The correct Table 1 can be found below.
Open Access This article is licensed under a Creative Commons Attribution 4.0 International License, which permits use, sharing, adaptation, distribution and reproduction in any medium or format, as long as you give appropriate credit to the original author(s) and the source, provide a link to the Creative Commons license, and indicate if changes were made. The images or other third party material in this article are included in the article's Creative Commons license, unless indicated otherwise in a credit line to the material. If material is not included in the article's Creative Commons license and your intended use is not permitted by statutory regulation or exceeds the permitted use, you will need to obtain permission directly from the copyright holder. To view a copy of this license, visit http://creativecommons. org/licenses/by/4.0/. 
Table 1 Baseline characteristics.

\begin{tabular}{|c|c|c|c|c|c|c|}
\hline & \multicolumn{2}{|c|}{ Usual care $(n=270)^{\mathrm{a}}$} & \multicolumn{2}{|c|}{ 10TT $(n=267)^{\mathrm{a}}$} & \multicolumn{2}{|c|}{ Total $(n=537)^{\mathrm{a}}$} \\
\hline & $N$ & $\%$ & $N$ & $\%$ & $N$ & $\%$ \\
\hline & Mean & S.d. & Mean & S.d. & Mean & S.d. \\
\hline & Median & IQR & Median & IQR & Median & IQR \\
\hline \multicolumn{7}{|l|}{ Socio-demographics } \\
\hline \multicolumn{7}{|l|}{ Age (years) } \\
\hline Median (IQR) & 60 & $48.9-67.1$ & 59.1 & $48.1-66.1$ & 59.4 & $48.7-66.8$ \\
\hline \multicolumn{7}{|l|}{ Gender } \\
\hline Male & 95 & $35.20 \%$ & 89 & $33.30 \%$ & 184 & $34.30 \%$ \\
\hline Female & 175 & $64.80 \%$ & 178 & $66.70 \%$ & 353 & $65.70 \%$ \\
\hline \multicolumn{7}{|l|}{ Ethnic origin $(n=534)$} \\
\hline White & 255 & $95.20 \%$ & 252 & $94.70 \%$ & 507 & $94.90 \%$ \\
\hline Black/Mixed & 5 & $1.90 \%$ & 5 & $1.90 \%$ & 10 & $1.90 \%$ \\
\hline Asian/Mixed & 6 & $2.20 \%$ & 6 & $2.30 \%$ & 12 & $2.20 \%$ \\
\hline Other & 2 & $0.80 \%$ & 3 & $1.10 \%$ & 5 & $0.90 \%$ \\
\hline \multicolumn{7}{|l|}{ Highest level of education $(n=505)$} \\
\hline No qualification/GCSE & 88 & $34.70 \%$ & 88 & $35.10 \%$ & 176 & $34.80 \%$ \\
\hline Vocational qualification/A-Level & 69 & $27.20 \%$ & 86 & $34.30 \%$ & 155 & $30.70 \%$ \\
\hline Degree or higher & 91 & $35.80 \%$ & 75 & $29.90 \%$ & 166 & $32.90 \%$ \\
\hline Other & 6 & $2.40 \%$ & 2 & $0.80 \%$ & 8 & $1.60 \%$ \\
\hline \multicolumn{7}{|l|}{ Deprivation (IMD) quintiles $(n=526)$} \\
\hline 1-Most deprived & 18 & $6.70 \%$ & 11 & $4.30 \%$ & 29 & $5.50 \%$ \\
\hline 2 & 54 & $20.20 \%$ & 45 & $17.40 \%$ & 99 & $18.80 \%$ \\
\hline 3 & 77 & $28.80 \%$ & 83 & $32.10 \%$ & 160 & $30.40 \%$ \\
\hline 4 & 66 & $24.70 \%$ & 49 & $18.90 \%$ & 115 & $21.90 \%$ \\
\hline 5-Least deprived & 52 & $19.50 \%$ & 71 & $27.40 \%$ & 123 & $23.40 \%$ \\
\hline \multicolumn{7}{|l|}{ Clinical } \\
\hline \multicolumn{7}{|l|}{ Weight $(n=536)(\mathrm{kg})$} \\
\hline Mean (S.d.) & 101.2 & -17.5 & 100.4 & -17 & 100.8 & -17.2 \\
\hline Median (IQR) & 98.6 & $88.4-110.7$ & 97.6 & $88.4-108.3$ & 98.4 & $88.4-109.7$ \\
\hline \multicolumn{7}{|l|}{ BMI $(n=536), \mathrm{kg} / \mathrm{m}^{2}$} \\
\hline Median (IQR) & 34.8 & $32.6-39.4$ & 35 & $32.6-38.7$ & 35 & $32.6-39.2$ \\
\hline \multicolumn{7}{|l|}{ Waist $(n=534), \mathrm{cm}$} \\
\hline Median (IQR) & 112 & $104-118$ & 111.3 & $103-120$ & 111.5 & $104-119$ \\
\hline \multicolumn{7}{|l|}{ Blood pressure (mmHg) } \\
\hline Systolic $(n=532)$, mean (s.d.) & 136.6 & -16.4 & 136.5 & -17.5 & 136.5 & -17 \\
\hline Diastolic $(n=532)$, mean (s.d.) & 81.4 & -10.1 & 81 & -10 & 81.2 & -10.1 \\
\hline \multicolumn{7}{|l|}{ Cholesterol, mmol/1 } \\
\hline Total $(n=473)$, mean (s.d.) & 5.2 & -1.1 & 5.2 & -1.2 & 5.2 & -1.2 \\
\hline LDL $(n=282)$, mean (s.d.) & 2.9 & -1 & 2.9 & -0.9 & 2.9 & -1 \\
\hline \multicolumn{7}{|l|}{ Glucose $(n=470), \mathrm{mmol} / \mathrm{l}$} \\
\hline Mean (s.d.) & 5.9 & -2.4 & 5.8 & -2.1 & 5.8 & -2.2 \\
\hline
\end{tabular}

$B M I$ Body Mass Index, GCSE General Certificate of Secondary Education, IMD Index of Multiple Deprivation, $I Q R$ interquartile range, $L D L$ Lowdensity lipoprotein.

${ }^{\mathrm{a}}$ Unless otherwise stated. 